\title{
The configuration determined by five generators of a quadric threefold
}

\author{
By L. M. Brown.
}

(Received 24th September, 1945. Read 2nd November, 1945.)

Through four generally placed lines in space of four dimensions there passes a doubly infinite system of quadric primals, but through five lines there pass in general no quadrics. It therefore follows that there must exist some special relationship between five lines in order that they may be generators of a quadric. This problem has been discussed by Richmond, ${ }^{1}$ who gives a condition which is in a restricted. sense an extension of Pascal's Theorem. The five lines being taken in order certain points may be obtained which lie in a space. In Section I we state Richmond's criterion and show that it is sufficient as well as necessary. Section II is concerned with the twelve spaces which arise if all the different possible orders of the lines are considered. They cut by pairs in six planes whose configuration is developed. In Section III other lines connected with the configuration are introduced. It is shown that by taking crossers of the lines of our original figure in a certain manner five further generators are obtained, and that the same entire configuration of generators arises whether we begin with the five original or the five final lines. Furthermore, though the twelve spaces analogous to Pascal lines obtained from the final five are new, yet the six planes, their intersections by pairs, and the configuration dependent from them, are the same as those constructed from the original five.

I.

Following Richmond's paper, take any five lines $a, \beta, \gamma, \delta, \epsilon$ on a quadric $\chi$ lying in a [4] $\sigma$. Then through $\sigma$ take a [5] $s$, and in $s$ a quadric $q$ through $\chi$. Through any line of $q$ goes one plane of each system of generating planes of $q$. Let $a, b, c, d, e$ be the planes of the first system through $\alpha, \beta, \gamma, \delta, \epsilon$ respectively. Any two planes of the same system meet in a point, so the five planes meet in ten points; call them $(a b),(a c), \ldots . .(d e)$. The three points $(a b),(a c),(b c)$ determine a plane we shall call $(a b c)$. It cuts $a, b, c$ in lines and is

1 Richmond, Proc. Camb. Phil. Soc., 10 (1899), 212. 
thus a generating plane of $q$ of the second system. The trace in $\sigma$ of $(a b c)$ is a line $(\alpha \beta \gamma)$ which cuts $\alpha, \beta, \gamma$ in the points $\alpha(\alpha \beta \gamma), \beta(\alpha \beta \gamma)$, $\gamma(a \beta \gamma) ; a(a \beta \gamma)$ is the trace in $\sigma$ of $a(a b c)$, i.e., of the line $(a b)(a c)$.

Richmond's criterion is as follows. Take the five generators in a definite cyclic order, say $a, \beta, \gamma, \delta, \epsilon$. Take the crosser of each line and its two neighbours in the order, $(\alpha \beta \gamma),(\beta \gamma \delta),(\gamma \delta \epsilon),(\delta \epsilon \alpha),(\epsilon \alpha \beta)$, and consider the five points where each crosser meets the middle line of the three it crosses, $a(\epsilon a \beta), \beta(\alpha \beta \gamma), \gamma(\beta \gamma \delta), \delta(\gamma \delta \epsilon), \epsilon(\delta \epsilon a)$. Then these five points lie in a space [a $\beta \gamma \delta \epsilon]$. (The order of the symbols in this bracket is material.) For they are the traces in $\sigma$ of the five lines $(e a)(a b)$, $(a b)(b c),(b c)(c d),(c d)(d e),(d e)(e a)$ in $s$, and these lie in the [4] [abcde] joining $(e a)(a b)(b c)(c d)(d e)$.

Conversely, take five lines $a, \beta, \gamma, \delta, \epsilon$ in a [4] $\sigma$ and let $(\alpha \beta \gamma)$ be the crosser of $\alpha, \beta, \gamma$ and define similarly $(\beta \gamma \delta),(\gamma \delta \epsilon),(\delta \in \alpha),(\epsilon \alpha \beta)$. Suppose that the five points $\alpha(\epsilon \alpha \beta), \beta(\alpha \beta \gamma), \gamma(\beta \gamma \delta), \delta(\gamma \delta \epsilon), \epsilon(\delta \epsilon a)$ lie in a [3] $[a \beta \gamma \delta \epsilon]$. Then we shall prove that $a, \beta, \gamma, \delta, \epsilon$ lie in a quadric $\chi$.

Through the [4] $\sigma$ take a [5] $s$ and in $s$ any plane $a$ through $a$. Join a general point $(a b)$ of $a$ to $\beta$ to obtain a plane $b$. The two [4]'s $a \gamma, b \gamma$ cut in a [3] through $\gamma$; take in it a general plane $c$ through $\gamma$; it cuts $a$ in a point $(a c)$ and $b$ in a point $(b c)$. The three [4]'s $a \epsilon, b \epsilon$, $c \epsilon$ cut in a plane $e$ through $\epsilon$ which cuts $a, b, c$ in three points $(a e)$, (be), (ce) respectively. And the three [4]'s $b \delta, c \delta, e \delta$ cut in a plane $d$ through $\delta$ which cuts $b, c, e$ in points $(b d),(c d),(d e)$ respectively. We have thus five planes $a, b, c, d, e$ such that by reason of their mode of construction every pair of them meet in a point except $a$ and $d$. We shall now prove that $a$ and $d$ meet in a point also.

Define $(e a b)$ as the plane joining (ae) (be) (ce). It cuts $e, a, b$ in lines so its section by $\sigma$ is $(\epsilon a \beta)$. Define similarly $(a b c)$ as $(a b)(a c)(b c)$, $(b c d)$ as $(b c)(b d)(c d)$ and $(c d e)$ as $(c d)(c e)(d e)$; their sections are $(a \beta \gamma)$, $(\beta \gamma \delta),(\gamma \delta \epsilon)$. Define [abcde] as the [4] $(e a)(a b)(b c)(c d)(d e)$. It contains the line $(e a)(a b)$ and thus the point $a(\epsilon a \beta)$. Similarly it contains $\beta(\alpha \beta \gamma), \gamma(\beta \gamma \delta), \delta(\gamma \delta \epsilon)$ and thus contains $[\alpha \beta \gamma \delta \epsilon]$. It therefore contains $\epsilon(\delta \epsilon a)$. The plane $e$ cuts [abcde] in the join of $(e d)(a e)$, so $\epsilon(\delta \epsilon a)$ lies in the join of $(e d)(a e)$. Hence $(\delta \in a)$ meets this join in $\epsilon(\delta \in a)$ and therefore $(\delta \epsilon a),(e d),(a e)$ lie in a plane we shall call $(d e a)$. Then (dea) contains $(e d)$ and $\delta(\delta \in \alpha)$ and both of these points lie in $d$, so (dea) cuts $d$ in a line $d(d e a)$. Similarly $(d e a)$ contains $(a e)$ and $a(\delta \epsilon a)$ which both lie in $a$, so (dea) cuts $a$ in a line $a(d e a)$. Then $d(d e a)$ and $a(d e a)$ meet in a point $(a d$ ) common to $a$ and $d$. 
So any two of the planes $a, b, c, d, e$ meet in a point. Then, as in Richmond's paper, there is a quadric $q$ through $a, b, c, d, e$. Cutting $q$ by $\sigma$, there is a quadric $\chi$ in $\sigma$ through $a, \beta, \gamma, \delta, \epsilon$.

So Richmond's criterion is both a necessary and a sufficient condition for $\alpha, \beta, \gamma, \delta, \epsilon$ to lie on a quadric.

$B$. Segre ${ }^{1}$ has recently proved this result by an entirely different method.

II.

There are twelve possible cyclic orders of the five lines $\alpha, \beta, \gamma, \delta, \epsilon$ and there consequently exist twelve spaces such as $[\alpha \beta \gamma \delta \epsilon]$. As Richmond observes, they combine naturally into six pairs, any space

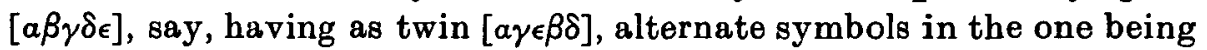
adjacent symbols in the other. We obtain in this way six planes, the intersections of twin spaces. Their mutual relations may be most conveniently discussed by developing further the configuration in the [5] $s$.

We have in $s$ five planes $a, b, c, d, e$, meeting by pairs in ten points $(a b), \ldots,(d e)$, and we have ten planes $(a b c), \ldots,(c d e),(a b c)$ being the join of $(a b)(a c)(b c)$. The [4] [ab] containing $a$ and $b$ contains $(a b),(a c),(b c)$ and thus contains $(a b c)$. So $(a b c)$ may be considered as the intersection of $[a b],[a c],[b c]$. The five points $(a b),(b c),(c d),(d e),(e a)$ lie in a [4] [abcde], and the five [4]'s [ab], [bc], [cd], [de], [ea] cut in a point (abcde).

Any two of the planes $(a b c), \ldots .,(c d e)$ meet in a point. Two which involve in their symbols only four letters meet in a point already known, e.g., ( $a b c)$ and $(a b d)$ meet in $(a b)$; but two planes involving all five letters meet in a new point, e.g., (abe), (cde) meet in a point we shall call $(A B, C D)$. (abe) cuts $e$ in the line $(a e)(b e)$ and (cde) cuts $e$ in $(c e)(d e)$ so $(A B, C D)$ is the intersection in $e$ of these lines. There are fifteen such points.

Now $(A B, D E),(A B, C E),(A B, C D)$ are by definition the intersections of $(c d e)$ with $(a b c),(a b d),(a b e)$ respectively: and $(a b c),(a b d)$, (abe) all lie in the [4] [ab]. Consequently $(A B, D E) ;(A B, C E)$, $(A B, C D)$ all lie in the line of intersection of $[a b]$ and (cde). Let us call this line $(A B)$. Our fifteen points $(A B, C D)$, etc., therefore lie on ten lines $(A B), \ldots,(D E)$; two of these lines meet if they have no common letter in their symbols. In the same way, if we define the [4]

1 B. Segre, Amer. Math. Monthly, 52 (1945), 119-131. 
[ $A B, C D]$ as the join of the two planes (abe) and (cde), the three [4]'s [ $A B, D E],[A B, C E],[A B, C D]$ all pass through the same [3] [AB], the join of $(a b)$ to (cde), and we have fifteen [4]'s passing through ten [3]'s.

Figure 1 shows the section of the configuration by the plane $e$. Figure 2 shows the fifteen points $(A B, C D)$, etc., and the ten lines $(A B)$, etc.
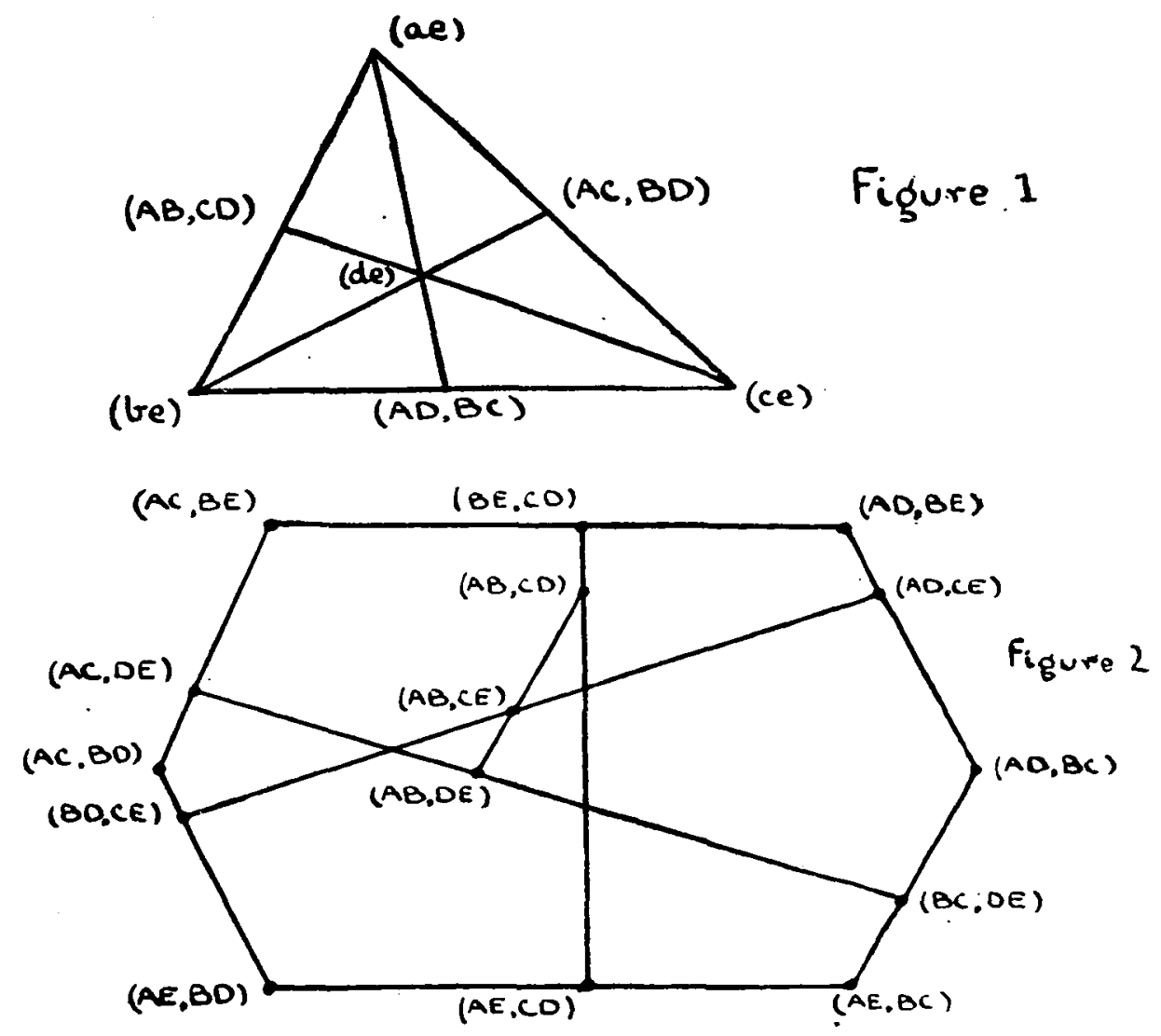

This configuration of ten lines has been discussed by Bath ${ }^{1}$ as the set of lines en a del Pezzo quintic surface. If we omit any line, $(A B)$ say, and the three lines $(C D),(C E),(D E)$ which meet it, the omitted lines lie in a [4], and the remaining six lines form a skew hexagon, $(A C)(B D)(A E)(B C)(A D)(B E)$. Furthermore the ten lines may be divided in six ways into two skew pentagons, each pentagon lying in a [4]; e.g., the pentagons $(A C)(B D)(C E)(A D)(B E)$ and $(A B)(C D)$

' Bath, Proc. Camb. Phil. Soc., 24 (1927-8), 48 aud 191. 
$(E A)(B C)(D E)$. Each line of one pentagon meets one line of the other, so these five points of intersection $(A C, D E),(A E, B D)$, $(A B, C E),(A D, B C),(B E, C D)$ lie in a [3]. This [3] is the intersection of the [4]'s [abcde], [acebd]. For $(A C, D E)$ lies in the line $(a b)(b c)$, $(B D, A E)$ lies in $(b c)(c d), \ldots,(B E, C D)$ lies in $(e a)(a b)$ so the [3] lies in the [4] $(a b)(b c)(c d)(d e)(e a)$ or [abcde], and similarly it lies in [acebd]. Dually $[A C, D E],[A E, B D],[A B, C E],[A D, B C],[B E, C D]$ pass through the line joining (abcde) to (acebd).

The ten lines form a convenient framework on which to hang the rest of the figure. The plane $a$ for example is $(B C, D E)(B D, C E)$ $(B E, C D)$ while $[a b]$ is the [4] which contains the three lines $(C D)$, $(C E),(D E)$ and so also their crosser $(A B) . \quad[A B, C D]$, being the tangent prime of $q$ at $(A B, C D)$, is determined by having to contain the lines $(A B),(C D)$ and the plane $e$. The [3], lying in this prime, which joins $e$ to $(C D)$ is the intersection of $[a e]$ and [be] because (CD) meets each of $a, b, e$.

It is convenient at this stage to introduce coordinates. Take the [4] [ab] as $x_{1}+x_{2}+x_{3}+x_{4}+x_{5}+x_{6}=0$ and take the six points $(A C, B E)$, etc., which do not lie in $[a b]$ as the base hexad, thus:

$$
\begin{aligned}
& (A C, B E) \equiv(1,0,0,0,0,0),(A C, B D) \equiv(0,1,0,0,0,0), \\
& (A E, B D) \equiv(0,0,1,0,0,0),(A E, B C) \equiv(0,0,0,1,0,0), \\
& (A D, B C) \equiv(0,0,0,0,1,0),(A D, B E) \equiv(0,0,0,0,0,1) .
\end{aligned}
$$

Then

$$
\begin{aligned}
& (A C, D E) \equiv(1,-1,0,0,0,0),(B D, C E) \equiv(0,1,-1,0,0,0), \\
& (A E, C D) \equiv(0,0,1,-1,0,0),(B C, D E) \equiv(0,0,0,1,-1,0), \\
& (A D, C E) \equiv(0,0,0,0,1,-1),(B E, C D) \equiv(-1,0,0,0,0,1), \\
& (A B, D E) \equiv(-1,1,0,-1,1,0),(A B, C E) \equiv(0,-1,1,0,-1,1), \\
& (A B, C D) \equiv(1,0,-1,1,0,-1) .
\end{aligned}
$$

The five planes $a, b, c, d, e$ are parametrically

$$
\begin{array}{cc}
a \equiv(\lambda, \mu,-\mu, \nu,-\nu,-\lambda), & b \equiv(\lambda,-\lambda, \mu,-\mu, \nu,-\nu), \\
c \equiv(-\lambda, \lambda, \mu,-\lambda, \lambda, \nu), & d \equiv(\mu,-\lambda, \lambda, \nu,-\lambda, \lambda), \\
e \equiv(\lambda, \mu,-\lambda, \lambda, \nu,-\lambda) .
\end{array}
$$

The quadric $q$ containing them is

$$
x_{1} x_{3}+x_{3} x_{5}+x_{5} x_{1}=x_{2} x_{4}+x_{4} x_{6}+x_{6} x_{2} .
$$

We shall write down the equations of the elements of the configuration we have already developed. For brevity we shall use the term "follows cyclically" when we replace each coordinate by the following coordinate in the cyclic order $x_{1}, x_{2}, x_{3}, x_{4}, x_{5}, x_{6}$. Thus the 
equation of the [4] [bc] is $x_{1}+x_{2}=0$; if wo say that [ad] follows cyclically from this we mean that its equation is $x_{2}+x_{3}=0$.

$[a b]$ is $x_{1}+x_{2}+x_{3}+x_{4}+x_{5}+x_{6}=0$,

$[b c]$ is $x_{1}+x_{2}=0$; [ad], [be], [ac], [bd], [ae] follow cyclically,

[ce] is $x_{1}=x_{4} ;[c d]$, [de] follow cyclically.

$(a b)$ is $(1,-1,1,-1,1,-1)$,

$(b c)$ is $(1,-1,-1,1,-1,1) ;(a d),(b e),(a c),(b d),(a e)$ follow cyclically, that is, $(a d)$ is $(1,1,-1,-1,1,-1)$, (be) is $(-1,1,1,-1,-1,1)$, and so on.

(ce) is $(-1,1,1,-1,1,1) ;(c d),(d e)$ follow cyclically.

(cde) is $x_{1}=x_{4}, x_{2}=x_{5}, x_{3}=x_{6}$,

(ade) is $x_{1}=x_{2}=-x_{3}=-x_{6} ;(b c e),(a c d),(b d e),(a c e),(b c d)$ follow cyclically,

$(a b d)$ is $x_{1}+x_{4}=x_{2}+x_{3}=x_{5}+x_{6} ;(a b e),(a b c)$ follow cyclically:

$[A D, B C]$ is $x_{1}+x_{3}=0 ;[A D, B E],[A C, B E],[A C, B D],[A E, B D]$,

$[A E, B C]$ follow cyclically, •

$[A D, C E]$ is $x_{1}+x_{2}+x_{3}+x_{4}=0 ; \quad[B E, C D],[A C, D E],[B D, C E]$, $[A E, C D],[B C, D E]$ follow cyclically,

$[A B, D E]$ is $x_{1}+x_{2}=x_{4}+x_{5} ;[A B, C E],[A B, C D]$ follow cyclically.

$[A B]$ is $x_{1}-x_{4}=x_{5}-x_{2}=x_{3}-x_{6}$;

$[B C]$ is $x_{1}+x_{3}=x_{2}+x_{6}=0$; $[A D],[B E],[A C],[B D],[A E]$ follow cyclically,

$[C E]$ is $x_{2}+x_{3}=x_{5}+x_{6}=-x_{1}-x_{4} ;[C D],[D E]$ follow cyclically.

[abedc] is $x_{3}+x_{4}+x_{5}-x_{1}=0 ; \quad[a b d e c],[a b d c e],[a b c d e],[a b c e d]$, [abecd] follow cyclically,

$[a e c b d]$ is $x_{3}+x_{4}+x_{5}+x_{1}=0 ;$ [adcbe], [adebc], [acebd], [acdbe], [aedbc] follow cyclically.

The twin [4]'s [abedc] and [aecbd] cut in a [3] which we shall call $s_{1}$; we similarly define $s_{2}, s_{3}, s_{4}, s_{5}, s_{6}$ as respectively the [3]'s [abdec] $[a d c b e],[a b d c e][a d e b c],[a b c d e][a c e b d],[a b c e d][a c d b e],[a b e c d][a e d b c]$. Their equations are

$$
\begin{array}{lll}
x_{1}=x_{3}+x_{4}+x_{5}=0, & x_{2}=x_{4}+x_{5}+x_{6}=0, & x_{3}=x_{5}+x_{6}+x_{1}=0, \\
x_{4}=x_{6}+x_{1}+x_{2}=0, & x_{5}=x_{1}+x_{2}+x_{3}=0, & x_{6}=x_{2}+x_{3}+x_{4}=0 .
\end{array}
$$

It is with the configuration of these six [3]'s and their duals we are chiefly concerned in this section.

Any two of the six [3]'s cut in a line; let $s_{1}$ and $s_{2}$ cut in a line $l_{12}$, etc. Then $l_{12}$ is parametrically $(0,0, \lambda+\mu,-\lambda,-\mu, \lambda+\mu)$ and $l_{23}, l_{34}, l_{45}, l_{56}, l_{16}$ follow cyclically, $l_{13}$ is $(0, \mu, 0, \lambda,-\lambda, \lambda)$ and $l_{24}, l_{35}, l_{46}$, 
$l_{15}, l_{26}$ follow cyclically; $l_{14}$ is $(0, \lambda, \mu, 0,-\mu,-\lambda)$ and $l_{2 \overline{5}}, l_{36}$ follow cyclically.

Dually, the point (abedc) is $(3,-1,-1,1,-1,-1)$ and $(a e c b d)$ is $(1,-1,1,1,1,-1)$ and the other points of the set of twelve follow cyclically, in the same order as their dual [4]'s. The join $l_{1}$ of the twin points (abedc) and (aecbd) is $(\lambda+\mu,-\lambda, \lambda-\mu, \lambda, \lambda-\mu,-\lambda)$ and $l_{2}, l_{3}, l_{4}, l_{5}, l_{6}$ follow cyclically. The [3] joining $l_{1}$ and $l_{2}$ we shall call $s_{12}$, etc.

$s_{12}$ is $x_{1}+x_{6}+x_{5}=x_{4}, x_{2}+x_{3}+x_{4}=x_{5}$ and $s_{23}, s_{34}, s_{45}, s_{56}, s_{16}$ follow cyclically,

$s_{13}$ is $x_{1}+2 x_{2}+x_{3}=x_{4}+x_{6}=0$ and $s_{24}, s_{85}, s_{48}, s_{15}, s_{26}$ follow cyclically,

$s_{14}$ is $x_{2}-x_{6}=x_{3}-x_{5}=0$ and $s_{25}, s_{36}$ follow cyclically.

Now $s_{1}$ is defined as the intersection of the [4]'s [abedc] and [aecbd], and this, as we saw earlier, is the join of the five points $(A B, C E),(A E, C D),(A D, B E),(A C, B D),(B C, D E)$. And in the same way $s_{2}$ is the join of the five points $(A B, C D),(A D, C E)$, $(A E, B C),(A C, B E),(B C, D E)$. Consequently $l_{12}$, the intersection of $s_{1}$ and $s_{2}$ passes through the point $(B C, D E)$. By a similar argument $l_{34}$ passes through $(B E, C D)$ and $l_{56}$ through $(B D, C E)$, so that the three lines $l_{12}, l_{34}, l_{56}$ all meet the plane $a$ in which these points lie. Dually the three [3]'s $s_{12}, s_{34}, s_{56}$ all meet the plane $a$ in lines. It is now easy to verify from their equations that these three lines are the intersections by pairs of these three [3]'s. In fact, $l_{12}$ is the intersection of $s_{34}, s_{56}, l_{34}$ that of $s_{56}, s_{12}, l_{56}$ that of $s_{12}, s_{34}$; consequently $s_{12}$ is the join of $l_{34}, l_{58}, s_{34}$ that of $l_{56}, l_{12}, s_{56}$ that of $l_{12}, l_{34}$.

We have thus, connected with the plane $a$, a triad of two-figure symbols.12, 34, 56. This is what Sylvester ${ }^{1}$ calls a syntheme. And there are in just the same way synthemes connected with each of the remaining four planes (each syntheme having similar properties to that described above) according to the scheme.

$$
\begin{array}{llllllllllllll}
a & 12 & 34 & 56 & & b & 16 & 23 & 45 & & c & 15 & 24 & 36 \\
& & & d & 14 & 26 & 35 & & e & 13 & 25 & 46 . & &
\end{array}
$$

This affords an example of a synthematic total of Sylvester.

The remaining. ten synthemes, or triads of two-figure symbols, we shall denote as follows

I Sylvester, Phil. Mag., 24 (1814), 285-296, Mathematical Papers, 1, 91-102. 


$\begin{array}{llllllll}A C & 13 & 26 & 45 & C D & 16 & 25 & 34 \\ B D & 13 & 24 & 56 & C E & 14 & 23 & 56 \\ A E & 16 & 24 & 35 & D E & 12 & 36 & 45 \\ B C & 12 & 35 & 46 & & & & \\ A D & 15 & 23 & 46 & A B & 14 & 25 & 36 \\ B E & 15 & 26 & 34 . & & & & \end{array}$

If we now arrange our symbols in the scheme

$\begin{array}{ccccc}a & A B & A C & A D & A E \\ A B & b & B C & B D & B E \\ A C & B C & c & C D & C E \\ A D & B D & C D & d & D E \\ A E & B E & C E & D E & e\end{array}$

the rows or columns give the remaining synthematic totals of Sylvester.

In the list of synthemes, the symbol 13, for example, belongs to the synthemes named $A C$ and $B D$. It is easy to verify from the equations given that $l_{13}$ passes through the point $(A C, B D)$ and lies in the [4]'s [ac] and [bd]; and dually that $s_{13}$ lies in the [4] $[A C, B D]$ and passes through the points $(a c)$ and $(b d)$. $s_{1}$ contains $(B C, D E),(A C, B D)$, $(A B, C E),(A D, B E),(A E, C D)$, which lie respectively in $l_{12}, l_{13}, l_{14}, l_{15}$, $l_{16}$; there is a dual result for $l_{1}$.

Furthermore, 13, 26, 45 being the syntheme $A C$, the three lines $l_{13}$, $l_{26}, l_{45}$ all lie in the same [3], which we shall call $s(A C)$. It passes through $(A C)$ and lies in [ac]. And dually, $s_{13}, s_{26}, s_{45}$ all pass through the same line, which we shall call $l(A C)$. It lies in the [3] [AC] and passes through $(a c)$. We have in this way ten lines and ten [3]'s.

$s(A B)$ is $x_{1}+x_{3}+x_{5}=x_{2}+x_{4}+x_{6}=0$.

$s(A C)$ is $x_{3}=x_{5}+x_{6}, x_{6}=x_{3}+x_{4} ; s(B D), s(A E), s(B C), s(A D)$, $s(B E)$ follow cyclically.

$s(C E)$ is $x_{2}+x_{3}+x_{4}+x_{5}+x_{6}=x_{1}+x_{2}+x_{3}+x_{5}+x_{6}=0 ; s(C D)$, $s(D E)$ follow cyclically.

$l(A B)$ is $(\lambda, \mu, \lambda, \mu, \lambda, \mu), l(A C)$ is $(2 \lambda-\mu, 2 \mu-\lambda,-3 \mu, 3 \lambda, 3 \mu,-3 \lambda)$, $l(C E)$ is $(\mu-2 \lambda, \lambda, \mu, \lambda-2 \mu, \mu, \lambda)$ and the other lines follow cyclically in the same order as their dual spaces above.

We observe that the whole configuration of lines $l$ and spaces $s$ may be constructed starting from $s_{1}, \ldots, s_{6}$. For $l_{12}, \ldots, l_{58}$ are their intersections, and $s_{12}, \ldots, s_{58}$ might be defined as the joins of certain pairs of these, as above, and then $l_{1}, \ldots, l_{6}$ appear as 
common lines of certain of $s_{12}, \ldots, s_{56}$; while $s(A C)$, etc., $l(A C)$, etc., are then defined as in the text.

The duality seen in the above work is of course a reciprocation in the quadric $q$.

Let us now see what happens when we cut this configuration by a [4] $\sigma$. We have as in I five lines $a, \beta, \gamma, \delta, \epsilon$, generators of a quadric $\chi$ in $\sigma$, and ten lines like $(\alpha \beta \gamma)$ the crosser of $a, \beta, \gamma$. There are ten [3]'s like $[a \beta]$, the join of $a$ and $\beta$. The join of the two lines $(\alpha \beta \epsilon)$, $(\gamma \delta \epsilon)$ is a [3] [AB, $\Gamma \Delta]$; there are fifteen such [3]'s. They pass by threes through ten planes; the three [3]'s $[\mathrm{AB}, \Gamma \Delta],[\mathrm{AB}, \Gamma \mathrm{E}],[\mathrm{AB}, \Delta \mathrm{E}]$ for instance pass through a plane [AB] in which lies the line $(\gamma \delta \epsilon)$.

The five points $a(a \beta \epsilon), \beta(a \beta \gamma), \gamma(\beta \gamma \delta), \delta(\gamma \delta \epsilon), \epsilon(a \delta \epsilon)$ all lie in a

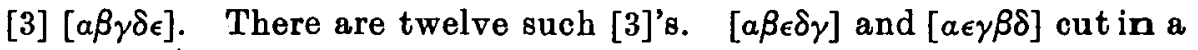
plane $\sigma_{1}$. There are six planes $\sigma_{1}, \ldots, \sigma_{6}$. Any two cut in a point, $\sigma_{1}$ and $\sigma_{2}$ cutting in $\lambda_{12}$; there are fifteen such points.

Ten triads of these determine ten planes $\sigma(\mathrm{A} \Gamma)$, etc., $\sigma(\mathrm{A} \Gamma)$ being the plane $\lambda_{13}, \lambda_{26}, \lambda_{45}$. It cuts $(\beta \delta \epsilon)$ in a point $(A \Gamma)$. There are ten such points (which lie on a normal elliptic quintic curve). (We show later that $(\mathrm{A} \Gamma)$ is the intersection of $(\beta \delta \epsilon)$ and the crosser of $(a \beta \gamma)$, $(a \gamma \delta),(a \gamma \epsilon)$.)

The five [3]'s [B $\Gamma, \Delta \mathrm{E}],[\mathrm{AE}, \Gamma \Delta],[\mathrm{A} \Gamma, \mathrm{B} \Delta],[\mathrm{AB}, \Gamma \mathrm{E}],[\mathrm{A} \Delta, \mathrm{B} \mathrm{E}]$ all pass through the same point $\lambda_{1}$; in the same way are defined $\lambda_{2}$, $\ldots \ldots, \lambda_{6}$. The four points $\lambda_{1}, \lambda_{2}, \lambda_{34}, \lambda_{56}$ all lie in the same plane $\sigma_{12}$. In this way are defined fifteen planes $\sigma_{12}, \ldots, \sigma_{56}$. The three planes $\sigma_{13}$, $\sigma_{26}, \sigma_{45}$ all meet in the same point $\lambda(\mathrm{A} \Gamma)$; there are ten such points.

In the [5] $s$, the [3] $s_{12}$ contains the points $(b c)$ and (de) and therefore their join, the common line of the [3]'s $b(5 d e), c(c d e), d(b c d)$, $e(b c e)$. Consequently $\sigma_{12}$ contains the common point of the planes $\beta(\beta \delta \epsilon)$, $\gamma(\gamma \delta \epsilon), \delta(\beta \gamma \delta), \epsilon(\beta \gamma \epsilon)$. This point is common to the four lines joining the pairs of points $\beta(\beta \gamma \delta) \delta(\beta \delta \epsilon), \beta(\beta \gamma \epsilon) \epsilon(\beta \delta \epsilon), \gamma(\beta \gamma \delta) \delta(\gamma \delta \epsilon)$, $\gamma(\beta \gamma \epsilon) \epsilon(\gamma \delta \epsilon)$, and is a well known point in the configuration of the four lines $\beta, \gamma, \delta, \epsilon$.

\section{III.}

Let us carry further the configuration in the [5] s. Define the plane $p(a b)$ as the join of the line $(A B)$ to the point $(a b)$. It is also the intersection of the [3] $[A B]$ and the [4] [ab]. $p(a b)$ and (cde) are the two generating planes of $q$ through the line $(A B)$. There are ten such plenes. As for their equations, 
$p(a b)$ is $x_{1}-x_{4}=x_{5}-x_{2}=x_{3}-x_{6}, x_{1}+x_{2}+x_{3}+x_{4}+x_{5}+x_{6}=0$, $p(a c)$ is $x_{3}=x_{4}=-x_{5}=-x_{6}$;

$p(b d), p(a e), p(b c), p(a d), p(b e)$ follow cyclically, $p(c e)$ is $x_{1}=x_{4}, x_{2}+x_{3}=x_{5}+x_{6}=-x_{1}-x_{4}$; $p(c d), p(d e)$ follow cyclically.

Any two of these planes meet in a point. Then the four points (ab), $p(a c) p(b c), p(a d) p(b d), p(a e) p(b e)$ all lie in a plane, which we shall call $(c d e)^{\prime}$. It may be obtained dually as the intersection of the [4]'s [ab], $[p(a c) p(b c)],[p(a d) p(b d)],[p(a e) p(b e)]$. There are ten such planes. These ten planes have the same mutual relations as the ten planes $(c d e), \ldots . .(a b c) ;(c d e)^{\prime},(b d \dot{e})^{\prime},(a d e)^{\prime}$ all meet in the same point $(d e)^{\prime}$, there being ten such points; $(d e)^{\prime},(c e)^{\prime},(b e)^{\prime},(a e)^{\prime}$ all lie in one plane $e^{\prime}$ and there are five such planes $a^{\prime}, b^{\prime}, c^{\prime}, d^{\prime}, e^{\prime}$. Similarly (cde)', $(b d e)^{\prime},(a d e)^{\prime}$ all lie in one [4] [de]' which joins $d^{\prime}$ and $e^{\prime}$. Since (cde)' passes through $(a b)$ and lies in $[a b]$ it cuts $(a b c),(a b d),(a b e)$ in lines.

These results are easy to prove analytically. For instance $p(a c) p(b c)$ is $(1,-1,-1,-1,1,1), p(a d) p(b d)$ is $(1,1,-1,-1,-1,1)$, $p(a e) p(b e)$ is $(1,1,1,-1,-1,-1)$ and the plane of these, $x_{1}+x_{4}=x_{2}+x_{5}=x_{3}+x_{6}=0$, contains $(a b)(1,-1,1,-1,1,-1)$. In this way

$(c d e)^{\prime}$ is $x_{1}+x_{4}=x_{2}+x_{5}=x_{3}+x_{6}=0$,

$(b d e)^{\prime}$ is $2 x_{2}+x_{3}+x_{4}=x_{4}+x_{5}=x_{5}+x_{6}+2 x_{1}=0$; $(a c e)^{\prime},(b d e)^{\prime},(\text { ade })^{\prime}$, $(b c e)^{\prime},(a c d)^{\prime}$ follow cyclically,

$(a b d)^{\prime}$ is $x_{1}-x_{4}=x_{2}-x_{3}=x_{5}-x_{6}=0 ;(a b e)^{\prime},(a b c)^{\prime}$ follow cyclically. $(a b)^{\prime}$ is $(1,1,1,1,1,1)$,

$(a c)^{\prime}$ is $(1,1,-3,3,3,-3) ;(b d)^{\prime},(a e)^{\prime},(b c)^{\prime},(a d)^{\prime},(b e)^{\prime}$ follow cyclically, $(c e)^{\prime}$ is $(3,-1,1,-3,1,-1) ;(c d)^{\prime},(d e)^{\prime}$ follow cyclically.

$[a b]^{\prime}$ is $x_{1}-x_{2}+x_{3}-x_{4}+x_{5}-x_{6}=0$,

$[a c]^{\prime}$ is $2 x_{3}+x_{4}=x_{5}+2 x_{6} ;[b d]^{\prime},[a e]^{\prime},[b c]^{\prime},[a d]^{\prime},[b e]^{\prime}$ follow cyclically, $[c e]^{\prime}$ is $x_{1}+2 x_{2}+2 x_{3}+x_{4}+2 x_{5}+2 x_{6}=0 ;[c d]^{\prime},[d e]^{\prime}$ follow cyclically. $a^{\prime}$ is $2 x_{1}+x_{2}=x_{3}+2 x_{4}, 2 x_{3}+x_{4}=x_{5}+2 x_{6}, 2 x_{6}+x_{6}=x_{1}+2 x_{2} ; b^{\prime}$ follows cyclically,

$c^{\prime}$ is $2 x_{3}+x_{4}=x_{5}+2 x_{6}, 2 x_{6}+x_{1}=x_{2}+2 x_{3}, 3 x_{1}+3 x_{2}+4 x_{3}+3 x_{4}+$ $3 x_{6}+4 x_{6}=0 ; d^{\prime}$ and $e^{\prime}$ follow cyclically.

Two planes $(a b e)^{\prime}$ and $(c d e)^{\prime}$ cut in a point $(A B, C D)^{\prime}$; there are fifteen such points lying on ten lines $(A B)^{\prime}, \ldots,(D E)^{\prime}$. We obtain dually fifteen [4]'s $[A B, C D]^{\prime}$, etc., passing through ten [3]'s $[A B]$, eto. 
$(A B, C D)^{\prime}$ is the point $(1,0,-1,-1,0,1)$. This lies in the line $l_{25}$, and since $l_{25}$ contains $(A B, C D)$, it may be considered as the join of $(A B, C D)$ and $(A B, C D)^{\prime}$. The three lines $l_{25}, l_{14}, l_{36}$ are therefore the joins of $(A B, C D),(A B, C E),(A B, D E)$ to $(A B, C D)^{\prime},(A B, C E)^{\prime}$, $(A B, D E)^{\prime}$. Consequently $s(A B)$ is the join of $(A B)$ to $(A B)^{\prime}$, for it contains $l_{2 b}, l_{14}, l_{36}$. Dually $s_{26}$ is the intersection of $[A B, C D]$ and $[A B, C D]^{\prime}$, and $l(A B)$ the intersection of $[A B]$ and $[A B]^{\prime}$. It follows that the same [3]'s $s_{1}, \ldots, s_{6}$ and lines $l_{1}, \ldots, l_{8}$ are obtained whether we begin with the five planes $a, b, c, d, e$ or the five planes $a^{\prime}, b^{\prime}, c^{\prime}, d^{\prime}, e^{\prime}$. Through $s_{1}$ for example there pass the following eight [4]'s :-

$$
\begin{aligned}
& {[a b e d c] \text { or } x_{3}+x_{4}+x_{5}-x_{1}=0,} \\
& {[a e c b d] \text { or } x_{3}+x_{4}+x_{5}+x_{1}=0,} \\
& (A B)(B E)(E D)(D C)(C A) \text { or } x_{3}+x_{4}+x_{5}=0, \\
& (A E)(E C)(C B)(B D)(D A) \text { or } x_{1}=0, \\
& {[a b e d c]^{\prime} x_{3}+x_{4}+x_{5}-3 x_{1}=0,} \\
& {[a e c b d]^{\prime} \text { or } 3\left(x_{3}+x_{4}+x_{5}\right)+x_{1}=0,} \\
& (A B)^{\prime}(B E)^{\prime}(E D)^{\prime}(D C)^{\prime}(C A)^{\prime} \text { or } x_{3}+x_{4}+x_{5}+2 x_{1}=0, \\
& (A E)^{\prime}(E C)^{\prime}(C B)^{\prime}(B D)^{\prime}(D A)^{\prime} \text { or } 2\left(x_{3}+x_{4}+x_{5}\right)-x_{1}=0 .
\end{aligned}
$$

If we now construct the ten planes $p(a b)^{\prime}, \ldots, p(d e)^{\prime}$, where $p(a b)^{\prime}$ is the join of $(a b)^{\prime}$ to $(A B)^{\prime}$ or the intersection of $[a b]^{\prime}$ and $[A B]^{\prime}$, we shall find that for instance the points $(a b)^{\prime}, p(a c)^{\prime} p(b c)^{\prime}$, $p(a d)^{\prime} p(b d)^{\prime}, p(a e)^{\prime} p(b e)^{\prime}$ all lie in the plane (cde). As an example of the algebra, $p(a c)^{\prime}$ is the plane $4 x_{1}+2 x_{2}+3 x_{3}+x_{4}=$ $8 x_{1}-2 x_{2}+3 \dot{x}_{3}+x_{6}=-2 x_{1}+2 x_{2}-x_{3}+x_{6}=0$ and $p(b c)^{\prime}$ is the plane $x_{1}+4 x_{4}+2 x_{5}+3 x_{6}=x_{2}+8 x_{4}-2 x_{5}+3 x_{6}=x_{3}-2 x_{4}+2 x_{5}-x_{6}=0$, and these cut in the point $(3,3,-7,3,3,-7)$ which lies in (cde) whose equations are $x_{1}-x_{4}=x_{2}-x_{5}=x_{3}-x_{6}=0$. Consequently, if we begin with $a^{\prime}, b^{\prime}, c^{\prime}, d^{\prime}, e^{\prime}$ and construct from them five planes in the same manner as $a^{\prime}, b^{\prime}, c^{\prime}, d^{\prime}, e^{\prime}$ were constructed from $a, b, c, d$, e, the five planes at which we arrive will be $a, b, c, d, e$. The process is involutory; in fact, if $t_{1}$ and $t_{2}$ are the two. roots of $t^{2}=t+1$, the six points $\left(t_{1}, 0,-2,2,-2,0\right), \quad\left(0, t_{2}, 0,-2,2,-2\right), \quad\left(-2,0, t_{1}, 0,-2,2\right)$, $\left(2,-2,0, t_{2}, 0,-2\right),\left(-2,2,-2,0, t_{1}, 0\right),\left(0,-2,2,-2,0, t_{2}\right)$ are coplanar, and so are the six points obtained by interchanging $t_{1}$ and $t_{2}$; then an harmonic involution of $s$ into itself with these two planes as base transforms each undashed element of our configuration into the corresponding dashed element, and vice versa.

Let us introduce two further sets of planes. Define $(a b, c d)$ as the plane joining $(A C, B D),(A D, B C),(A B, C D)^{\prime}$; there are fifteen 
like this. And define $(a b, c d)^{\prime}$ as the plane joining $(A C, B D)^{\prime}$, $(A D, B C)^{\prime},(A B, C D)$; there are fifteen like this. The three points $(A C, B D),(A D, B C),(A B, C D)^{\prime}$ are $(0,1,0,0,0,0),(0,0,0,0,1,0)$, $(1,0,-1,-1,0,1)$, so the equations of $(a b, c d)$ are $x_{1}=-x_{3}=-x_{4}=x_{6}$. So $(a b, c d)$ contains the points $p(a c) p(a d), p(b c) p(b d), p(a c) p(b c)$, $p(a d) p(b d)$ whose coordinates are respectively $(1,1,-1,-1,1,1)$, $(-1,1,1,1,1,-1),(1,-1,-1,-1,1,1),(1,1,-1,-1,-1,1)$. Thus $(a b, c d)$ cuts each of $p(a c), p(a d), p(b c), p(b d)$ in lines; it also cuts $e$ in a line, for $e$ contains $(A C, B D)$ and $(A D, B C)$. Moreover (cde)' contains $p(a c) p(b c)$ and $p(a d) p(b d)$ by its definition, so $(a b, c d)$ cuts (cde)' in a line, and similarly it cuts $(a b e)^{\prime}$ in a line. It also meets $(a c, b d)^{\prime}$ and $(a d, b c)^{\prime}$ in lines; in fact, $(a b, c d)$ and $(a c, b d)^{\prime}$ cut in the line joining $(A B, C D)^{\prime}$ to $(A C, B D)$.

It is worth observing that if the points of the quadric $q$ in [5] are mapped in the classical manner on to the lines of a [3], then to the planes $a, b, c, d, e$ correspond five points $A, B, C, D, E$ and to the planes $a^{\prime}, b^{\prime}, c^{\prime}, d^{\prime}, e^{\prime}$ correspond five planes $A^{\prime}, B^{\prime}, C^{\prime}, D^{\prime}, E^{\prime}$. The configuration of these points and planes has been discussed by Burnside. ${ }^{1}$

We shall now return to the section of this configuration by the [4] $\sigma$. The planes $p(a b)$, etc., cut $\sigma$ in ten lines $\sigma(a \beta), \ldots, \varpi(\delta \epsilon)$. $\varpi(\alpha \beta)$ for example meets the four lines $(\alpha \beta \gamma),(\alpha \beta \delta),(\alpha \beta \epsilon),(\gamma \delta \epsilon)$, meeting the last in the point $(\mathrm{AB})$. The crossers of any three of $(\alpha \beta \gamma), \ldots$, $(\gamma \delta \epsilon)$ are now given among the lines $a, \beta, \gamma, \delta, \epsilon, \varpi(\alpha \beta), \ldots \ldots, \varpi(\delta \epsilon)$ (of course some sets of three are cospacial, e.g., $(\alpha \beta \gamma),(\alpha \beta \delta),(\alpha \beta \epsilon)$ lie in

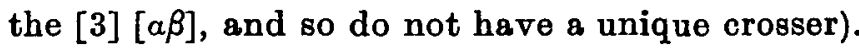

There are fifteen lines $(\alpha \beta, \gamma \delta), \ldots,(\beta \gamma, \delta \epsilon)$, where $(\beta \gamma, \delta \epsilon)$, for instance, meets $\alpha, \varpi(\beta \delta), \varpi(\beta \epsilon), \varpi(\gamma \delta), \varpi(\gamma \epsilon)$.

Then $(\alpha \beta \gamma)^{\prime}$ may be defined as the crosser of $(\alpha \beta, \delta \epsilon),(\alpha \gamma, \delta \epsilon),(\beta \gamma, \delta \epsilon)$. It also meets $(a \delta \epsilon),(\beta \delta \epsilon),(\gamma \delta \epsilon)$, though it cannot be defined as their crosser, since these three lines lie in $[\delta \epsilon]$. There are ten lines like this, $(\alpha \beta \gamma)^{\prime}, \ldots,(\gamma \delta \epsilon)^{\prime}$.

There is a line $\varpi(\alpha \beta)^{\prime}$ meeting $(\alpha \beta \gamma)^{\prime},(\alpha \beta \delta)^{\prime},(\alpha \beta \epsilon)^{\prime},(\gamma \delta \epsilon)^{\prime}$. There are ten such lines $\varpi(\alpha \beta)^{\prime}, \ldots, \varpi(\delta \epsilon)^{\prime}$.

There is a line $a^{\prime}$ meeting $(\alpha \beta \gamma)^{\prime},(\alpha \beta \delta)^{\prime},(\alpha \beta \epsilon)^{\prime},(\alpha \gamma \delta)^{\prime},(\alpha \gamma \epsilon)^{\prime},(\alpha \delta \epsilon)^{\prime}$. There are five such lines $a^{\prime}, \beta^{\prime}, \gamma^{\prime}, \delta^{\prime}, \epsilon^{\prime}$.

1 Burnside, Proc. Camb. Phil. Soc., 16 (1911), 418 ; see also Baker, Principles of Geometry, 3 (1923), 168. 
There is a line $(\alpha \beta, \gamma \delta)^{\prime}$ meeting $a^{\prime}, \varpi(a \gamma)^{\prime}, \varpi(a \delta)^{\prime}, \varpi(\beta \gamma)^{\prime}, \varpi(\beta \delta)^{\prime}$, $(\alpha \beta \epsilon),(\gamma \delta \epsilon),(\alpha \gamma, \beta \delta),(\alpha \delta, \beta \gamma)$. There are fifteen such lines.

If from $\alpha^{\prime}, \beta^{\prime}, \gamma^{\prime}, \delta^{\prime}, \epsilon^{\prime}$ we construct five lines in the same manner as $a^{\prime}, \beta^{\prime}, \gamma^{\prime}, \delta^{\prime}, \epsilon^{\prime}$ are obtained from $a, \beta, \gamma, \delta, \epsilon$, then the five lines obtained are $a, \beta, \gamma, \delta, \epsilon$.

All these lines are generators of the quadric $\chi$. Though we have written down their relations immediately from the section of $s$ by $\sigma$, it is clear that many of these may easily be obtained directly without having recourse to a higher dimension.

If from $\alpha^{\prime}, \beta^{\prime}, \gamma^{\prime}, \delta^{\prime}, \epsilon^{\prime}$ are constructed points and planes $\lambda_{12}, \lambda(\mathrm{AB})$, $\lambda_{1}, \sigma_{12}, \sigma(\mathrm{AB}), \sigma_{1}$, etc., as was done from $a, \beta, \gamma, \delta, \epsilon$, then the same system of points and planes is obtained as before.

Since in $s$ an harmonic inversion in two planes $r_{1}, r_{2}$ leaves invariant the spaces $s_{12}, s(A B), s_{1}$, etc., it follows that each of these spaces meets both $r_{1}$ and $r_{2}$ in lines. Consequently in $\sigma$ there are two lines $\rho_{1}, \rho_{2}$ each meeting all of the fifteen planes $\sigma_{12}$, etc., the ten planes $\sigma(\mathrm{AB})$, etc., and the six planes $\sigma_{1}, \ldots, \sigma_{6}$.

The Mathematical Institute, The University, Edinburgh. 